\title{
Reduction of Magnet Eddy Current Loss in PMSM by using Partially Magnet Segment Method
}

\author{
Youhua Wang ${ }^{1,2}$, Jinguang $\mathrm{Ma}^{1,2}$, Chengcheng Liu ${ }^{1,2^{*}}$, Gang $\mathrm{Lei}^{3}$, Youguang $\mathrm{Guo}^{3}$, Jianguo Zhu ${ }^{4}$ \\ ${ }^{1}$ State Key Laboratory of Reliability and Intelligence of Electrical Equipment, Hebei University of Technology, Tianjin \\ 300130, China \\ ${ }^{2}$ Province-Ministry Joint Key Laboratory of EFEAR, Hebei University of Technology, Tianjin 300130, China \\ ${ }^{3}$ School of Electrical and Data Engineering, University of Technology Sydney, NSW 2007, Australia \\ ${ }^{4}$ School of Electrical and Information Engineering, University of Sydney, NSW 2007, Sydney, Australia
}

\begin{abstract}
Compared with traditional induction machine and direct current (DC) machine, permanent magnet synchronous machine (PMSM) holds the merits of higher torque ability, efficiency and etc., when high magnetic co-energy sintered NdFeB magnet is used. However for high frequency operation, the resulted eddy current loss by permanent magnet (PM) is very high and this kind of loss can bring the PM with high temperature rise, moreover it will make the PM face the risk of irreversible demagnetization. To reduce PM eddy current loss, complete magnet segmentation (CMS) is an effective method, however taking this kind of method will increase manufacturing cost and reduce mechanical robustness of PMSM. Thus, a partially magnet segmentation method was proposed in the past. In this paper, a new annular partial segmentation method is proposed for PM eddy current loss reduction, namely single side annular partial segment (SSAPS) and double side annular partial segment (DSAPS). Considering that the additional process on the PM will reduce the mechanical robust of the $P M$ and electromagnetic performance of machine, both the electromagnetic performance and the mechanical strength of PM has been analyzed, based on 3D finite element method (FEM). It can be found that if the proposed new annular partial segmentation (APS) method is adopted, then the eddy current loss in PM can be reduced greatly while the mechanica comparing with the traditional partial magnet segmentation method.
\end{abstract}

Index Terms--Eddy current loss, permanent magnet, 3D finite element methods, partial segmentation, mechanical strength.

\section{INTRODUCTION}

$I^{N}$ $\mathrm{N}$ recent years, permanent magnet synchronous machine (PMSM) have become more and more popular and gaining strong research interests in both academic and industry field [13]. Compared with traditional induction machine and direct current (DC) machine, PMSM can have higher power density and efficiency, due to the adopted permanent magnet (PM) can produce very strong excited magnetic field, especially when sintered NdFeB magnet is used [4-5]. However, for high speed or high frequency operation state, the resulted eddy current loss in PM cannot be ignored, since this kind of loss can not only decrease the machine efficiency but also bring the PM with high temperature rise. Moreover, it can make the PM machine face the risk of magnet irreversible demagnetization, especially, for PMSM with fractional slot concentrated winding (FSCW) configuration where more high order spatial harmonics are existed compared with the PMSM with distributed windings [68].

In past years, some different methods were proposed for reducing the PM eddy current loss, e.g. optimized stator/rotor shape, improved winding design, and magnet design. For the optimized stator/rotor shape design and improved winding design, the reduction of PM eddy current loss is mainly resulted because the spatial harmonics has been changed, thus it can

Manuscript received November 5, 2018;

Corresponding author: C.C.Liu (e-mail: 2016020@hebut.edu.cn). only be used for the special kind of PMSM [9-12]. Though the PM eddy current loss can be reduced greatly and even more than half by using above method as compared with the initial design, the improved magnet design are still employed for the PMSM as the necessary of further PM loss reduction. Specifically, for the magnet design the magnet needs to be divided completely then glued together namely complete magnet segmentation (CMS), which will bring the PM machine with high manufacturing cost and low mechanical robustness. In [13], a partial magnet segmentation method was proposed, where the magnet is not divided completely and thus the mechanical robustness of the magnet can be guaranteed. The partial magnet segmentation can be composed of single side partial segmentation (SSPS) configuration and double side partial segmentation (DSPS) configuration.

In this paper, a novel magnet segmentation method is proposed for the PM eddy current loss reduction of PMSM with FSCW, namely annular partial segmentation (APS) method, which can be composed of single side annular partial segment (SSAPS) configuration and double side annular partial segment (DSAPS) configuration. For fair comparison, both the electromagnetic performance and mechanical performance of CMS, SSPS, DSPS, SSAPS, and DSAPS are optimized and compared based on the Maxwell 3D and ANSYS Workbench. Finally, it can be conclude that the proposed novel SSAPS can achieve better performance. 


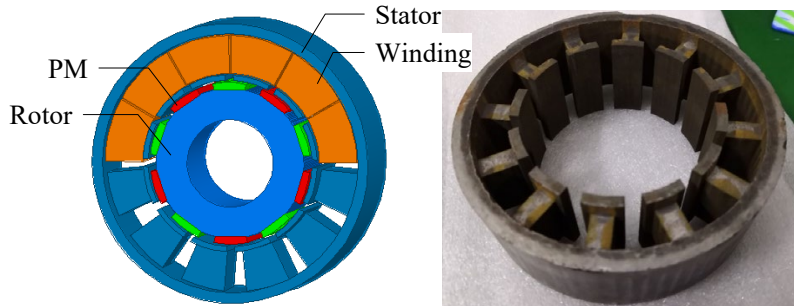

Fig. 1.Main electromagnetic part of PMSM-FSCW

\section{Magnet SEgmentation Analysis}

\section{A. PMSM-FSCW}

In this paper, a PMSM-FSCW with 12 stator slots and 10 magnet poles is used for case study. The stator core is made by using the soft magnetic composite (SMC) material, and rotor core is made by using the silicon steels. As the adopted SMC core can bring the PMSM-FSCW with 3-D magnetic flux ability, the stator core has be designed with extended stator yoke in axial direction and stator teeth in both axial direction and circumferential direction and no end winding outside to form very compact structure, as shown in Fig. 1. The main dimensions and the parameters of PMSM-FSCW are tabulated in Table 1. As shown, the rated power of PMSM-FSCW is about $4 \mathrm{~kW}$ and the maximum speed is about $12000 \mathrm{rpm}$.

TABLE I MAIN DIMENSIONS OF MACHINE
\begin{tabular}{|c|c|c|c|}
\hline Parameter name & Value & Parameter name & Value \\
\hline $\begin{array}{c}\text { Phase peak } \\
\text { voltage }\end{array}$ & $150 \mathrm{~V}$ & N coils & 9 \\
\hline Rated speed & $3000 \mathrm{rpm}$ & Magnet thickness(MT) & $3.80 \mathrm{~mm}$ \\
\hline Maximum speed & $12000 \mathrm{rpm}$ & Magnet width(MW) & $15.50 \mathrm{~mm}$ \\
\hline Rated power & $4 \mathrm{~kW}$ & Magnet density & $7400 \mathrm{~kg} / \mathrm{m}^{3}$ \\
\hline Slot number & 12 & Magnet Young is Modulus & $1.6 \times 10^{11} \mathrm{~Pa}$ \\
\hline Pole number & 10 & Magnet Poisson is Ratio & 0.26 \\
\hline $\begin{array}{c}\text { Stator outer } \\
\text { radius }\end{array}$ & $67.31 \mathrm{~mm}$ & Magnet Tensile strength & $80 \mathrm{MPa}$ \\
\hline $\begin{array}{c}\text { Rotor outer } \\
\text { radius }\end{array}$ & $41.65 \mathrm{~mm}$ & Remnant flux density & $1.2 \mathrm{~T}$ \\
\hline $\begin{array}{c}\text { Motor axial } \\
\text { length(MAL) }\end{array}$ & $41.91 \mathrm{~mm}$ & $\begin{array}{c}\text { Magnet electrical } \\
\text { conductivity }\end{array}$ & $\begin{array}{c}625000(\mathrm{~S} / \\
\mathrm{m})\end{array}$ \\
\hline
\end{tabular}

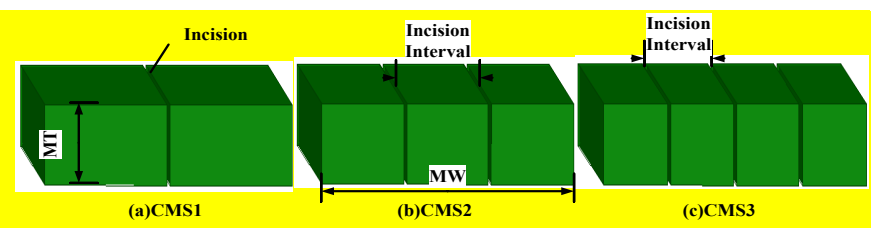

Fig. 2. Configuration of CMS with different division number.

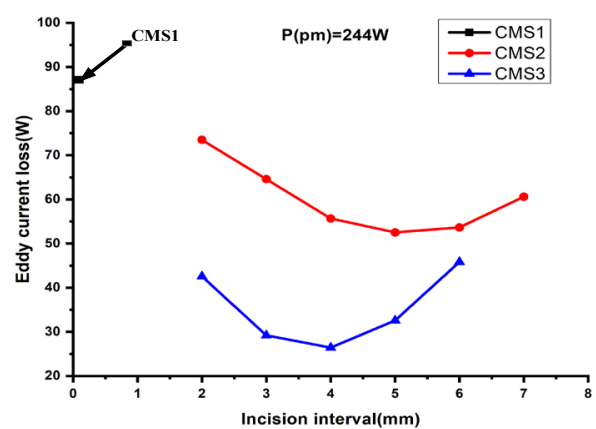

Fig. 3. PM eddy current loss in CMS with different incision interval.

\section{B. Complete magnet segmentation (CMS)}

CMS is the most popular way to reduce PM eddy current loss in PMSM, as shown in Fig. 2, where CMS1, CMS2, and CMS3 represents the division number of $\mathrm{PM}$ is 2,3 , and 4 respectively. As shown, in CMS2 and CMS3, the incision interval can be varied and thus the resulted PM eddy current loop will be changed. By using the 3-D finite element method (FEM), PM eddy current loss of CMS1, CMS2 and CMS3 with different incision interval have been calculated, as shown in Fig. 3. For CMS1, the best way is to divide the PM into two equal parts, thus the incision interval is zero. It can be found that when the incision interval equals $5 \mathrm{~mm}$ and $4 \mathrm{~mm}$, then the eddy current loss for both CMS2 and CMS3 can achieve lowest value. For CMS1, CMS2 it shows that the magnet needs to be divided evenly while for CMS3 the PM needs to be divided unevenly then the PM eddy current loss can achieve minimum value. The PM eddy current loss for the entire magnet is about $244 \mathrm{~W}$, for CMS1 it is about $87 \mathrm{~W}$, for optimized CMS2 it is about $55 \mathrm{~W}$ while for optimized CMS3 it is about $28 \mathrm{~W}$.

CMS can make PMSM-FSCW achieve very low PM eddy current loss, however this method is quite complex since the magnets needs to be insulated and retighten, which will increase manufacturing cost and reduce mechanical robustness of the magnet.

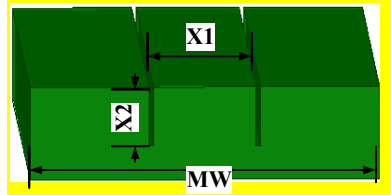

(a) SSPS

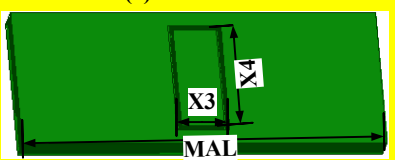

(c) SSAPS

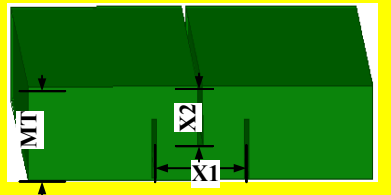

(b) DSPS

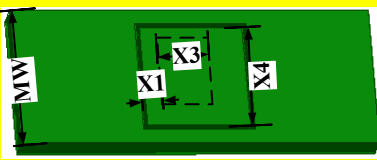

(d) DSAPS
Fig. 4. Configuration of different partially magnet segmentation method

\section{Partial Magnet Segmentation (PMS)}

Compared with CMS, PMS is another method to reduce the PM eddy current loss. With PM is segmented only partially, PM can keep with a completely part and no additional manufacturing cost required. While, the PM eddy current loss will be higher than that in CMS. In this section, a new (APS) is proposed with two configurations namely SSAPS and DSAPS, their performance was compared with the SSPS and DSPS. Fig. 4 shows the main structure of SSPS, DSPS, SSAPS, and DSAPS. In SSAPS and DSAPS, the incision shape is rectangular. Moreover for DSAPS the upper surface incision and bottom surface incision are located in different position. For SSPS, two parameters are defined for optimization, X1 represents the incision interval, $\mathrm{X} 2$ represents incision depth, as the magnet is partial segmented X2 is lower than the magnet thickness. As for SSAPS and DSAPS, X3 is defined as the incision distance in axial direction, $\mathrm{X} 4$ is defined as the incision distance in circumferential direction. The design of above parameters in both SSPS, DSPS, SSAPS and DSAPS need to ensure the PM as a complete part while with low eddy current loss and manufacturing cost.

By using 3D FEM, the eddy current loss of PMSM-FSCW 
with above magnet configurations can be calculated. As shown in Fig. 5(a), with the X2 increase the eddy current decreases greatly. Considering the integrity of magnet, $\mathrm{X} 2$ is determined not over than $2.5 \mathrm{~mm}$ which is the $65 \%$ of the magnet thickness. Fig. 5(b) shows the PM eddy current loss with the variation of $\mathrm{X} 2$ in SSPS and DSPS. It can be seen that the X1 equals about $4 \mathrm{~mm}$ then the SSPS can achieve the minimum PM eddy current loss, as for the DSPS, the $\mathrm{X} 1$ equals about $2 \mathrm{~mm}$ then the PM eddy current loss can achieve the minimum value. The main reason is that the resistance in the closed loop can achieve the maximum value. While, low incision interval value will bring the DSPS with low mechanical robustness. As for SSAPS, its PM eddy current loss will be decreased with the X4 increases and when $\mathrm{X} 4$ equals $13 \mathrm{~mm}$ then it can achieve the minimum value when $\mathrm{X} 3$ equals $6 \mathrm{~mm}$, as shown in Fig. 5(c). Fig. 5(d) shows the effect of X1 and X3 on PM eddy current loss, it can be seen that when axial span equals about $6 \mathrm{~mm}$ and incision interval equals about $3 \mathrm{~mm}$ then the PM eddy current loss can reach minimum value. The optimized dimension for these four magnets is tabulated in Table II.

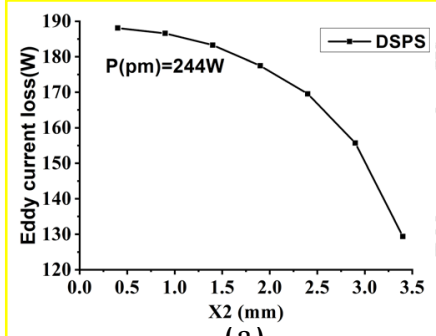

(a)

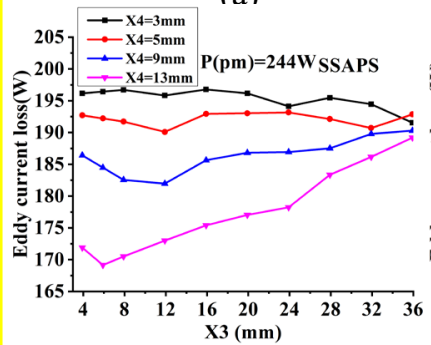

(c)

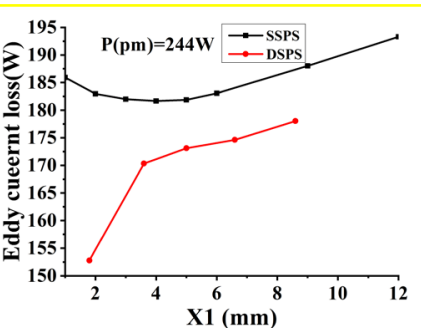

(b)

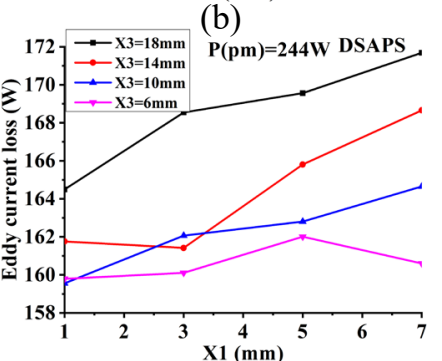

(d)
Fig. 5. Variation of eddy current losses versus incision parameters, (a) Incision depth (X2) in DSPS, (b) Incision interval (X1) in SSPS and DSPS, (c) Incision axial span (X3) and circumferential span (X4) in SSAPS, and (d) Incision interval (X1)and axial span (X3) in DSAPS

TABLE II Main Parameter for SSPS, DSPS, SSAPS and DSAPS for Achieving Lowest PM Eddy Current Loss

\begin{tabular}{|c|c|c|c|c|}
\hline & $\begin{array}{c}\text { Incision } \\
\text { depth(X2)(mm) }\end{array}$ & $\begin{array}{c}\text { Incision } \\
\text { interval(X1)(mm) }\end{array}$ & $\begin{array}{c}\text { Circumferential } \\
\text { span }(\mathrm{X} 4)(\mathrm{mm})\end{array}$ & $\begin{array}{c}\text { Axial } \\
\text { span(X3)(mm) }\end{array}$ \\
\hline SSPS & 2.5 & 4 & 0 & 0 \\
\hline DSPS & 2.5 & 4 & 0 & 0 \\
\hline SSAPS & 2.5 & 0 & 13 & 6 \\
\hline DSAPS & 2.5 & 3 & 13 & 6 \\
\hline
\end{tabular}

Fig. 6 shows the eddy current path and distribution on the surface of initial PM and PM with four different PMS configurations with optimized parameters tabulated in Table II. As shown, the eddy current distribution on the surface of initial PM and SSPS, DSPS, SSAPS, DSAPS are similar, the main difference is that the eddy current started on the PM with PMS configuration needs to penetrate the narrow part which were made on PM to form a closed eddy current loop, and for different PMS configuration the narrow part is different. This narrow part plays main role to increase the resistance of PM and thus the eddy current can be reduced. Fig. 7 shows the eddy current loss distribution on the initial PM, and PM with four different PMS configurations. As shown the eddy current loss distributed on the surface of initial PM is the highest one and for the PM with four different PMS configurations the maximum eddy current loss density occurred on the narrow part of PM.

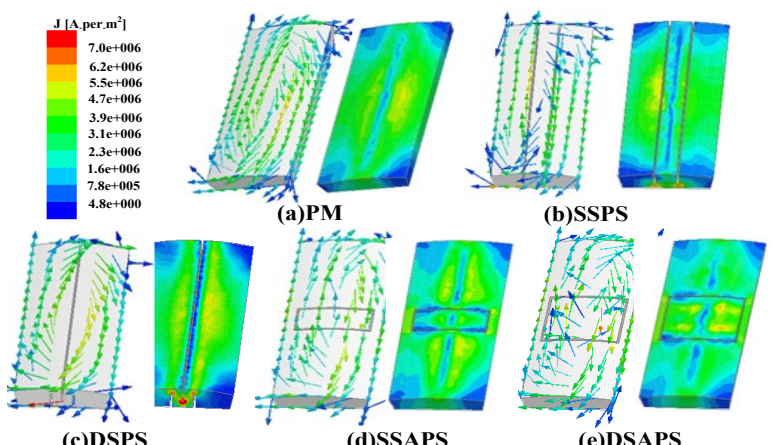

Fig. 6. Eddy current path and distribution on the surface of initial PM and PM with four different PMS configurations.

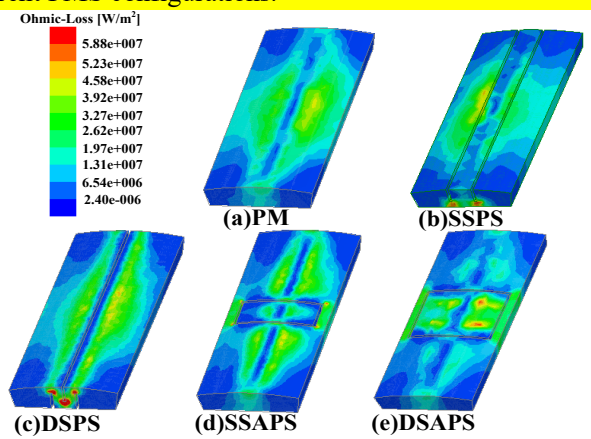

Fig. 7. Eddy current loss distribution on the surface of initial PM, and PM with four different PMS configurations.

\section{Mechanical Strength Analysis}

In section II, it can be seen that with different PMS configurations, the PM eddy current loss in PMSM-FSCW can be reduced effectively. However with different parameter design, the mechanical robustness of these PMs is quite different. Therefore it is necessary to analyze the mechanical strength of above different partially segmentation method. Table II tabulates the optimized parameters of above four PMs. By using the ANSYS Workbench, the total deformation and equivalent stress of these PMs with the rotate speed of 12000 $\mathrm{rpm}$ has been calculated. In the calculation of structural deformation and equivalent stress, the electromagnetic force produced on the PM is considered.

Fig. 8 shows the structural deformation of these four PMs resulted by large tangential centrifugal force and electromagnetic force. It can be seen that the maximum deformation occurs at the incision edge on the upper surface of PM and the structural deformation on the bottom surface of PM is quite small, due to the incision is existed on the upper surface 
of the PM which is far away from the axis and the bottom surface of PM is attached on the rotor surface. As shown these four PMs have the similar structural deformation

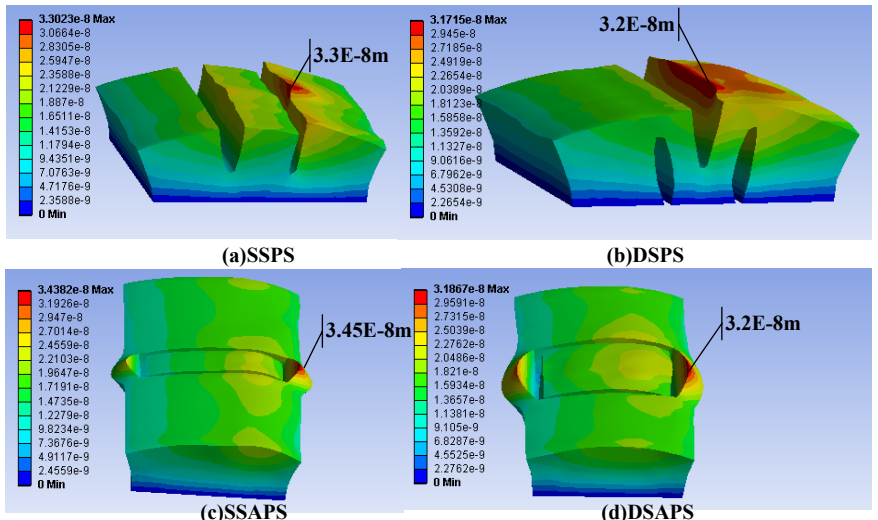

Fig. 8. Total deformation analysis of PM with four PMS configuration

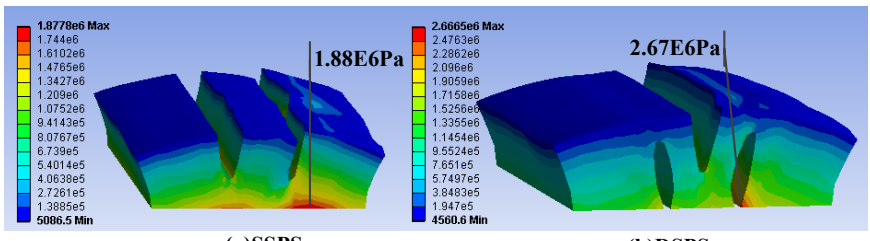
(a)SSPS

(b)DSPS

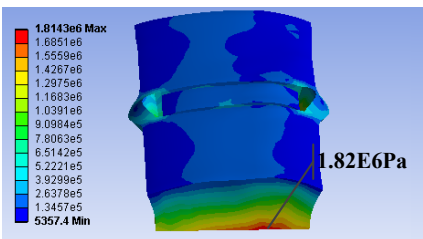

(c)SSAPS

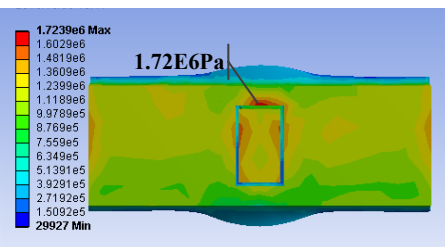

(d)DSAPS
Fig. 9. Equivalent stress analysis of four models

Fig. 9 shows the equivalent stress of these four PMs resulted by large tangential centrifugal force and electromagnetic force. it can be seen that the maximum equivalent stress occurs at incision edge and PM bottom surface. Because the PM is attached on the surface of rotor core, the maximum equivalent stress occurs on the bottom surface of PM. Among these four PMs, the PM with DSPS has the highest equivalent stress of $2.67 \times 10^{6} \mathrm{~Pa}$, which is lower than the tensile strength of used $\mathrm{PM}$ of $8 \times 10^{7} \mathrm{~Pa}$. Therefore, the resulted equivalent stress by the centrifugal force will not destroy the mechanical structure of the PM.

\section{OVERALl PERformance COMPARISON}

During the process of PM eddy current reduction, the electromagnetic performance of PMSM-FSCW with the new magnet design will be affected. In this section, the influence of magnet segmentation to PMSM-FSCW is analyzed. Fig. 10 shows the no load air gap flux density distribution. It can be seen that the fundamental order of no load air gap flux density of PMSM-FSCW with initial PM design is the maximum one, and the difference of that with other PM design is not high. The PMSM-FSCW with SSAPS design has the second high air gap flux density. Fig.11 shows the electromagnetic torque comparison it can be seen that the difference among these six machines are quite low. The overall comparison is listed in Table III. It can be found that the proposed SSAPS, the mechanical robust kept the similar to the PM with no additional process, but the PM eddy current reduced about $30 \%$ and with only torque reduced about $1.3 \%$.

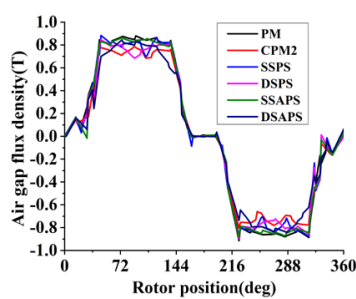

(a)

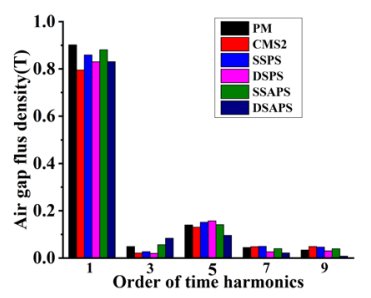

(b)
Fig. 10.(a) No load air gap flux density distribution of above six PM configurations. (b)major harmonic no load air gap flux density distribution

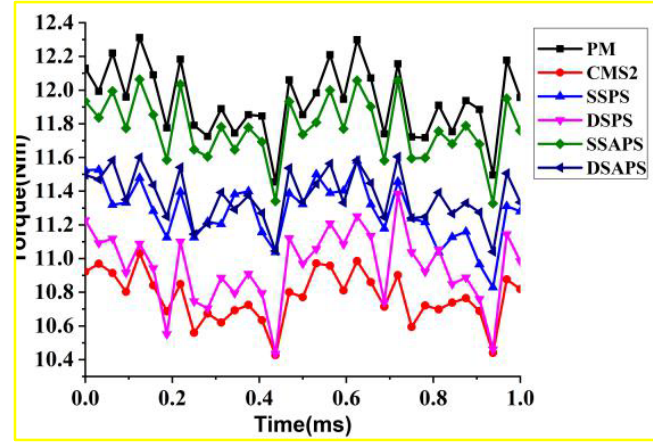

Fig. 11 Torque distribution of above six PM configurations.

\begin{tabular}{|c|c|c|c|c|c|}
\hline \multicolumn{1}{|c|}{ Table III Overall Performance Comparison } \\
\hline $\begin{array}{c}\text { Eddy } \\
\text { Current } \\
\text { Loss(W) }\end{array}$ & $\begin{array}{c}\text { \% Eddy } \\
\text { Current } \\
\text { Loss } \\
\text { Reduction }\end{array}$ & Torque(Nm) & $\begin{array}{c}\text { \% Torque } \\
\text { Reduction }\end{array}$ & $\begin{array}{c}\text { Equivalent } \\
\text { Stress(MPa) }\end{array}$ \\
\hline PM & 244 & 0 & 11.93 & 0 & 1.77 \\
\hline CMS2 & 52.5 & 78 & 10.77 & 9.7 & 1.65 \\
\hline SSPS & 183 & 25 & 11.29 & 5.4 & 1.88 \\
\hline DSPS & 170 & 30.3 & 10.97 & 8.1 & 2.67 \\
\hline SSAPS & 168 & 31.1 & 11.77 & 1.3 & 1.82 \\
\hline DSAPS & 160 & 34.4 & 11.37 & 4.7 & 1.72 \\
\hline
\end{tabular}

\section{CONCLUSION}

In this paper, PM eddy current loss reduction for permanent magnet synchronous machine (PMSM) is studied. Especially a new annual partially segmentation method is proposed and compared with the complete magnet segmentation (CMS) and traditional partial magnet segmentation method. By using the 3D finite element method (FEM), both the electromagnetic performance and mechanical performance of the permanent magnet synchronous machine-fractional slot concentrated winding (PMSM-FSCW) with above different permanent magnet (PM) are calculated. It can be concluded, first for the CMS when the division number is 2,3 then it needs to be divided evenly for achieving the minimum PM eddy current loss while for CMS3 it needs to be divided unevenly. Second for the partial magnet segmentation the proposed annual partial 
magnet segmentation are better than the traditional partial magnet segmentation. Third for the overall performance it can be found that with the new annual partial magnet segmentation the PM can kept similar equivalent stress if compared with the PM with no additional processing.

\section{REFERENCE}

[1] A. M. EL-Refaie et al., "Advanced High-Power-Density Interior Permanent Magnet Motor for Traction Applications," IEEE Trans. Magn., vol. 50, no. 5, pp. 3235-3248, Sept.-Oct. 2014.

[2] Z. Q. Zhu and D. Howe, "Electrical Machines and Drives for Electric, Hybrid, and Fuel Cell Vehicles," Proceeding of the IEEE, vol. 95, no. 4, pp. 746-765, April 2007.

[3] T. Fukami, Y. Ueno and K. Shima, "Magnet Arrangement in Novel FluxModulating Synchronous Machines With Permanent Magnet Excitation," IEEE Trans. Magn., vol. 51, no. 11, pp. 1-4, Nov. 2015.

[4] M. Park, H. Kim, Y. Choi, J. Hong and J. Lee, "Characteristics of IPMSM According to Rotor Design Considering Nonlinearity of Permanent Magnet," IEEE Trans. Magn., vol. 52, no. 3, pp. 1-4, March 2016.

[5] T. F. Chan, L. L. Lai and Lie-Tong Yan, "Performance of a three-phase $\mathrm{AC}$ generator with inset $\mathrm{NdFeB}$ permanent-magnet rotor," IEEE Trans. Energy Convers., vol. 19, no. 1, pp. 88-94, March 2004..

[6] G. Choi and T. M. Jahns, "Reduction of Eddy-Current Losses in Fractional-Slot Concentrated-Winding Synchronous PM Machines," IEEE Trans. Magn. vol. 52, no. 7, pp. 1-4, July 2016.

[7] S. Chaithongsuk, N. Takorabet and S. Kreuawan, "Reduction of EddyCurrent Losses in Fractional-Slot Concentrated-Winding Synchronous PM Motors," IEEE Trans. Magn. vol. 51, no. 3, pp. 1-4, March 2015.

[8] L. Wu, R. Qu and D. Li, "Reduction of Rotor Eddy-Current Losses for Surface PM Machines With Fractional Slot Concentrated Windings and Retaining Sleeve," IEEE Trans. Magn., vol. 50, no. 11, pp. 1-4, Nov. 2014.

[9] K. Kim, K. Kim, H. J. Kim and J. Lee, "Demagnetization Analysis of Permanent Magnets According to Rotor Types of Interior Permanent Magnet Synchronous Motor," IEEE Trans. Magn., vol. 45, no. 6, pp. 2799-2802, June 2009.

[10] K. Yamazaki, Y. Fukushima and M. Sato, "Loss Analysis of PermanentMagnet Motors With Concentrated Windings-Variation of Magnet Eddy-Current Loss Due to Stator and Rotor Shapes," IEEE Trans. Ind. Appl., vol. 45, no. 4, pp. 1334-1342, July/Aug. 2009.

[11] S. Y. Oh et al., "Design of IPMSM Rotor Shape for Magnet Eddy-Current Loss Reduction," IEEE Trans. Magn., vol. 50, no. 2, pp. 841-844, Feb. 2014.

[12] K. Yamazaki, M. Shina, Y. Kanou, M. Miwa and J. Hagiwara, "Effect of Eddy Current Loss Reduction by Segmentation of Magnets in Synchronous Motors: Difference Between Interior and Surface Types," IEEE Trans. Magn., vol. 45, no. 10, pp. 4756-4759, Oct. 2009.

[13] D. A. Wills and M. J. Kamper, "Reducing PM eddy current rotor losses by partial magnet and rotor yoke segmentation," XIX Int. conf. on elec. Mach. 2010, pp. 1-6. 